\title{
Mindfulness y síntomas de depresión en adolescentes mexicanos estudiantes de bachillerato
}

\author{
Mindfulness and Symptoms of Depression in \\ Mexican Adolescents High School Students
}

\author{
Marcela Veytia-López ${ }^{1}$, \\ Rosalinda Guadarrama Guadarrama², \\ Octavio Márquez-Mendoza ${ }^{3}$, \\ Rocio J. Fajardo Gómez ${ }^{4}$ \\ Universidad Autónoma del Estado de México, México
}

\begin{abstract}
Resumen. El objetivo de esta investigación fue identificar la asociación entre síntomas depresivos y niveles de mindfulness en adolescentes mexicanos estudiantes de bachillerato. Se realizó un estudio transversal con 930 estudiantes de bachillerato entre 15 y 19 años de edad. Se aplicaron los instrumentos CESD (síntomas de depresión) y MAAS (mindfulness) y se encontró un $17.3 \%$ de prevalencia de síntomas depresivos. Además, el 55.2\% de los participantes presentaron niveles bajos de mindfulness; los adolescentes con síntomas depresivos presentaron mayores niveles de mindfulness. La asociación entre síntomas de depresión y mindfulness fue negativa $(\mathrm{r}=-0.53 ;<.001)$. Conclusión: Impulsar el estudio sobre el rasgo de atención plena o mindfulness como un factor psicológico positivo porque se demuestra su efecto reductor en síntomas de depresión.
\end{abstract}

Palabras clave. Mindfulness, depresión, adolescentes, estudiantes, bachillerato.

Abstract. The objective of this research was to identify the relation between depressive symptoms and mindfulness levels in mexican adolescents high school students. A cross-sectional survey was conducted with 930 baccalaureate students between 15 and 19 years old was performed. The instruments, CESD (depressive symptoms) and MAAS (mindfulness) were applied, finding a $17.3 \%$ prevalence of depressive symptoms, furthermore, $55.2 \%$ of participants had low levels of mindfulness, and also adolescents with depressive symptoms have higher levels of mindfulness. The relation between depression symptoms and mindfulness was negative $(r=-0.53 ;<.001)$. Conclusion: Study the feature of full awareness or mindfulness as a positive psychological factor because it's reducing effect is demonstrated in psychological symptoms such as depression.

Keywords. Mindfulness, depression, adolescents, students, high school.

\footnotetext{
${ }^{1}$ Marcela Veytia-López. Universidad Autónoma del Estado de México, México. Dirección postal: Mariano Abasolo 202 Col. Cuauhtémoc. CP50130. Toluca, México E-mail: mveytial@uaemex.mx

${ }^{2}$ Rosalinda Guadarrama Guadarrama. Universidad Autónoma del Estado de México, México. E-mail: rossygma@hotmail.com

${ }^{3}$ Octavio Marquez-Mendoza. Universidad Autónoma del Estado de México, México. E-mail: octavio-mar@hotmail.com

${ }^{4}$ Rocío J. Fajardo Gómez. Universidad Autónoma del Estado de México, México. E-mail: chio.fajardogomez@gmail.com
}

\section{(ब) $\Theta \Theta$}

Esta obra está bajo una licencia de Creative Commons Reconocimiento-NoComercial-SinObraDerivada 4.0 Internacional. 


\section{Introducción}

La Psicología Cognitiva se ha encargado de sentar las bases que sustentan los procesos y estructuras mentales de las personas como sujetos activos que construyen sus propios conocimientos. Desde esta premisa, la conducta se describe y se explica en términos del procesamiento que se realiza la persona sobre las representaciones mentales encontradas entre el estímulo y la respuesta observable de esta (Rivière, 1991). Entre los aspectos psicológicos que se han estudiado en relación con la cognición-emoción está el mindfulness o atención plena (MDF), relacionado con la conciencia de la persona en un momento determinado. Contiene el manejo y reconocimiento de estímulos cognitivos y emocionales (Sánchez y Araya-Vargas; 2014), enfocándose en los aspectos positivos que tiene ante síntomas psicológicos.

La atención plena o mindfulness se ha definido como un proceso psicológico donde emerge un estado mental en el que la persona es altamente consciente y enfoca la realidad del momento presente (Williams, Teasdale, Segal \& Kabat-Zinn, 2007). Tiene como premisa básica el uso intencional de la atención con la finalidad de hacer conscientes las reacciones automáticas que contribuyen al desequilibrio emocional y la conducta disfuncional (Miró et al., 2011). Para Germer (2011), el mindfulness consiste en el proceso psicológico de enfocar la atención en un objeto determinado, sin juzgar ni dejarse llevar por pensamientos o reacciones emotivas. Este incrementa la capacidad de responder habilidosamente a los procesos mentales que contribuyen al desarrollo de malestares emocionales y es un proceso con disposición duradera o de estado fluctuante.

De acuerdo con Van den Hurk et al. (2011), el mindfulness es un constructo que está relacionado con la personalidad, refiriendo que las habilidades de atención plena actúan como factores de mediación entre la práctica de mindfulness y la personalidad, lo cual requiere un proceso psicológico complejo, que implica la conciencia de estar alerta y presente para producir distinciones emocionales y cognitivas.
El mindfulness ha sido ampliamente estudiado, relacionando su eficacia y efectividad con diversos trastornos (Marchand, 2016) y condiciones médicas diversas como estrés, ansiedad, dolor crónico, cáncer y depresión (Sánchez \& Araya-Vargas, 2014). Además, se ha mostrado que el MDF se ha asociado negativamente con síntomas psicológicos tales como depresión, ansiedad, conducta antisocial y consumo de drogas (Calvete, Sampedro \& Izaskun, 2014).

En investigaciones relacionadas con el tema de la depresión desde el modelo cognitivo, se ha observado que pensamientos vagos están asociados con síntomas depresivos, refiriendo que el propio pensamiento negativo causa síntomas depresivos (Linares, Estévez, Soler \& Cebolla, 2016). Los pensamientos recurrentes se han correlacionado de forma positiva con la disforia; el humor negativo también ha referido contribuir a la elevación de pensamientos no conscientes (Smallwood, Fitzgerald, Miles \& Philips, 2009). Esta evidencia implica que un estado de ánimo negativo activa el pensamiento negativo y aumenta la posibilidad de recaída depresiva (Marchand, 2016).

Se ha documentado que una de las poblaciones más vulnerables a la depresión son los adolescentes, ya que se encuentran en la etapa de desarrollo donde han de enfrentarse tanto a los riesgos sociales representados en situaciones de su entorno (Giménez, Vázquez \& Hervás, 2010). También, se encuentran con diversidad de cambios que experimentan en la transición de la adolescencia hacia la adultez, además de innumerables transformaciones a nivel biopsicosociocultural, que llevan al adolescente a interferir en su toma de decisiones y resolución de problemas (Cortés, Aguilar, Medina, Toledo \& Echemendia, 2010). Lo anterior influye en el inicio y desarrollo de conductas de riesgo y de algún trastorno mental, frente a lo cual, se ha comenzado a reportar que la edad de inicio de la mayoría de los trastornos psiquiátricos se ubica en las primeras décadas de la vida; cuando se presenta antes de los 18 años, el curso de la enfermedad puede llegar a ser más crónico y de mayor duración (Berenzon, Lara, Robles \& Medina-Mora, 2013). 
En México, algunas prevalencias de depresión en población adolescente indican que el $27.5 \%$ de los adultos diagnosticados con depresión presentaron episodios o síntomas depresivos antes de los 18 años de edad (González-Forteza, Hermosillo, Vacio-Muro, Peralta \& Wagner, 2015). Asimismo, la Encuesta Mexicana de Salud Mental Adolescente reportó que el $10.5 \%$ de adolescentes, entre los 12 y 17 años de edad, presentaron algún tipo de trastorno afectivo como depresión mayor (Benjet et al., 2009). En relación con los estudios realizados con estudiantes de bachillerato, se encontró que el 15.6\% de adolescentes presentaron síntomas de depresión (Veytia-López, GonzálezArratia, Andrade \& Oudhof, 2012).

En cuanto a las diferencias por género sobre síntomas depresivos González-Forteza et al. (2015), reportaron que en la adolescencia aumentan los trastornos depresivos en ambos sexos; sin embargo, se acentúan más en las mujeres. Estas diferencias comienzan a surgir a partir de los 15 años de edad y se detienen a los 18 años aproximadamente (Hankin, Mermelstein \& Roesch, 2007). Además, pueden deberse a una mayor vulnerabilidad cognitiva a la depresión y, en las mujeres, a una reactividad a estresores sociales (Shih, Abela \& Starrs, 2009).

Con base en los antecedentes señalados previamente, esta investigación tiene como objetivo identificar la asociación entre niveles de mindfulness con los síntomas de depresión en estudiantes adolescentes mexicanos de bachillerato, denotando que se tiene como marco de referencia a la Psicología Positiva, por considerar a la salud mental como algo más que la ausencia de trastornos mentales (Vázquez \& Hervás, 2008). Esta sugiere que los recursos psicológicos positivos, tales como fortalezas y virtudes humanas (Remor, 2008), operan como factor protector ante las adversidades. Al mismo tiempo, demuestra científicamente que las personas con mayor bienestar psicológico tienen mejores apreciaciones de sí mismos, un mejor dominio del entorno y mejores habilidades sociales (Castro, 2010), por lo que se necesita el desarrollo y uso de nuevas estrategias como el mindfulness para lograr ese objetivo deseable.

\section{Método}

\section{Participantes}

Participaron 930 adolescentes, estudiantes de bachillerato, de dos preparatorias públicas de la Ciudad de Toluca de México. Las edades eran entre 14 y 19 años $(M=15.75, D T=0.87)$; de los cuales el $59.68 \%$ eran mujeres y el $40.32 \%$ hombres. El $35.9 \%$ de los participantes cursaba el primer semestre, el 80.9\% estudiaba en el turno matutino y el $94.8 \%$ de los adolescentes vivían con sus padres.

\section{Instrumentos}

Para evaluar mindfulness se utilizó la Escala de Conciencia de Atención Consciente (MAAS-A; Brown, West, Loverich \& Biegel, 2011) en su versión al español, traducida por Barajas y Garra (2014). Consiste en 14 ítems que evalúan el rasgo de conciencia plena. Mide la frecuencia de la atención abierta y receptividad a los acontecimientos y experiencias que tienen lugar. Las respuestas oscilan entre 1 (casi nunca) y 6 (casi siempre), por lo que el rango de puntuación total oscila entre 14 y 84. Las puntuaciones de los ítems se recodificaron de modo que las puntuaciones más altas representaran un mayor grado de atención plena.

Las puntuaciones se obtienen a partir de la media aritmética del total de ítems, indicando que a mayores puntuaciones existe un mayor estado mindfulness. El punto de corte para este estudio fue de 33 para los hombres y de 34 para las mujeres. La consistencia interna (alpha de Cronbach) de la escala ha sido satisfactoria en estudios realizados a nivel internacional con adolescentes, puntuando entre .80 a .90 (Barros, Kozasa, Souza \& Ronzani, 2015; Calvete et al., 2014; Nava et al., 2015). Para esta investigación se obtuvo una consistencia interna $\alpha=.89$.

Para evaluar los síntomas de depresión, se utilizó la Escala de Depresión del Centro de Estudios Epidemiológicos (CES-D; Radloff, 1977) en su versión al español revisada por González-Forteza, Jiménez-Tapia, Ramos-Lira y Wagner (CES-D-R; 2008) la cual está integrada por 20 ítems. Es una escala de tamizaje para detectar probables casos de depresión. La CES-D- R 
evalúa cuatro factores (afecto depresivo, afecto positivo, disminución psicomotora y dificultades interpersonales) y un factor de depresión general de segundo orden.

Se realizó un ajuste de la escala de respuesta para que diera información de la presencia de síntomas en las últimas dos semanas, con cinco opciones de respuesta: $0=$ ningún día; $1=$ de 1 a 2 dias; $2=$ de 3 a 4 dias; $3=$ de 5 a 7 días y $4=$ de 8 a 14 días. Las puntuaciones de la escala varían entre 0 y 60 puntos, indicando que, a mayor puntuación, más síntomas de depresión. El punto de corte para este estudio fue de 24 puntos para los hombres y de 34 para las mujeres. La consistencia interna de la escala ha sido satisfactoria en estudios con adolescentes mexicanos reportando un alpha de Cronbach desde .82 a .93 (González-Forteza et al, 2008; Veytia-López et al, 2012). En esta investigación se obtuvo el coeficiente de confiabilidad alpha de Cronbach total de .87 .

\section{Procedimiento}

Esta investigación forma parte del proyecto Factores Psicológicos Positivos como Elementos Protectores de la Depresión en Estudiantes Adolescentes y fue aprobado por el comité de ética del Centro de Investigación en Ciencias Médicas-UAEM. La aplicación de los instrumentos fue de manera grupal dentro del horario de clases, en un tiempo aproximado de 30 minutos. Participaron tres aplicadores estudiantes de psicología previamente capacitados para el manejo de los instrumentos y la retroalimentación a los participantes, de modo que pudieran aclarar las dudas que se presentaran durante la aplicación. Se entregaron cartas de consentimiento informado para sus padres y para los adolescentes. Los alumnos que hicieron la devolución de las cartas autorizadas fueron los que participaron en la investigación.

\section{Análisis Estadístico}

Se realizaron análisis descriptivos, medias y desviación estándar cuando las variables fueron continuas y porcentajes cuando las variables fueron categóricas. Para identificar la relación entre mindfulness y síntomas de depresión se empleó una correlación de Spearman. Para las diferencias por sexo se llevó a cabo una " $\mathrm{t}$ " de Student o U de Mann Whitney para muestras independientes. Con un nivel de significancia preestablecido de $<.05$.

\section{Resultados}

En la tabla 1 se muestran los resultados de las características sociodemográficas de los 930 adolescentes encuestados. Para el diagnóstico de depresión, se encontró que el $17.3 \%$ presentan síntomas, observando la misma prevalencia tanto en mujeres como en hombres. También, se pudo observar que las mujeres presentaron un $55.3 \%$ de bajos niveles de mindfulness; en tanto, los hombres obtuvieron un $54.9 \%$.

$\mathrm{Al}$ analizar las variables con respecto al sexo, utilizando la prueba U de Mann Whitney, en la tabla 2 se observa que, en la muestra total, existen diferencias estadísticamente significativas entre los síntomas de depresión y mindfulness, siendo mayor el puntaje de mindfulness en hombres y de depresión en mujeres. El puntaje de depresión es menor en los hombres con depresión, en tanto el puntaje de las mujeres se mantiene. Con respecto al mindfulness, cuando se obtuvieron puntajes altos se presentaron menores puntajes de depresión en ambos sexos.

Realizando un análisis de $\chi^{2}$ se encontró una asociación estadísticamente significativa entre las variables que se midieron con el CESD y el MAAS tanto para el grupo de hombres como en el de las mujeres, es decir, que cuando existen puntajes altos de síntomas de depresión y puntajes bajos de mindfulness estos se asocian (ver tabla 3).

Para examinar la relación entre la presencia de mindfulness y los síntomas de depresión, se realizó un análisis de correlación de Spearman (tabla 4). Se encontró que existe una correlación negativa moderada y estadísticamente significativa entre estas dos variables $(\mathrm{r}=.53 ; p<.001)$. Lo anterior indica que a mayores puntajes de depresión, menores puntajes de mindfulness o viceversa. 
Tabla 1

Características de la población, nivel de mindfulness y sintomas de depresión de acuerdo al sexo (n=930)

\begin{tabular}{|c|c|c|c|}
\hline & $\begin{array}{c}\text { Mujeres } \\
555(59.68)\end{array}$ & $\begin{array}{c}\text { Hombres } \\
375 \text { (40.32) }\end{array}$ & $\begin{array}{c}\text { Total } \\
930(100)\end{array}$ \\
\hline Edad (años) ${ }^{1}$ & $15.68 \pm 0.82$ & $15.85 \pm 0.94$ & $15.75 \pm 0.87$ \\
\hline \multicolumn{4}{|l|}{ Turno } \\
\hline Matutino & $466(83.9)$ & $287(76.6)$ & $753(80.9)$ \\
\hline Vespertino & 89 (16.1) & $88(23.4)$ & $177(19.1)$ \\
\hline \multicolumn{4}{|l|}{ Semestre } \\
\hline $1^{\circ}$ & $197(35.5)$ & $137(36.5)$ & $334(35.9)$ \\
\hline $3^{\circ}$ & $287(51.7)$ & $173(46.1)$ & $460(49.5)$ \\
\hline $5^{\circ}$ & $71(12.8)$ & $65(17.4)$ & $136(14.6)$ \\
\hline \multicolumn{4}{|l|}{ Estado Civil } \\
\hline Casado & $3(0.5)$ & $13(3.4)$ & $16(4.2)$ \\
\hline Soltero & $552(99.4)$ & $362(96.5)$ & $914(98.3)$ \\
\hline \multicolumn{4}{|l|}{ Con quién vive } \\
\hline Padres & $529(95.3)$ & $353(94.1)$ & $882(94.8)$ \\
\hline Otros familiares & $23(4.1)$ & $19(5.1)$ & $42(4.5)$ \\
\hline Solo & $3(0.5)$ & $3(0.8)$ & $6(0.6)$ \\
\hline \multicolumn{4}{|l|}{ Síntomas depresivos } \\
\hline Con síntomas & $96(17.3)$ & $65(17.3)$ & $161(17.3)$ \\
\hline Sin síntomas & $459(82.7)$ & $310(82.7)$ & $769(82.7)$ \\
\hline \multicolumn{4}{|l|}{ Mindfulness } \\
\hline Alto & $248(44.7)$ & $169(45.1)$ & $417(44.8)$ \\
\hline Bajo & 307 (55.3) & $206(54.9)$ & $513(55.2)$ \\
\hline
\end{tabular}

Nota. Los datos se presentan en porcentaje; ${ }^{1}$ Media \pm Desviación Típica.

\section{Discusión}

En la presente investigación, el objetivo principal fue identificar la asociación entre síntomas depresivos y niveles de mindfulness en adolescentes mexicanos estudiantes de bachillerato. Los resultados obtenidos indican que el $17.3 \%$ de los adolescentes que participaron en este estudio presentaron SD donde, además. se observó una prevalencia igual de síntomas depresivos entre hombres y mujeres, a diferencia de los estudios realizados por González-Forteza et al. (2015), quienes encontraron que las mujeres presentaron mayores síntomas depresivos a diferencia de los hombres, esto debido tanto a aspectos biológicos como psicológicos y sociales.

Los resultados aquí presentados son novedosos por encontrar las mismas cifras de prevalencia de síntomas de 
Tabla 2.

Comparación de las variables depresión y niveles de mindfulness por sexo para la muestra general y con depresión

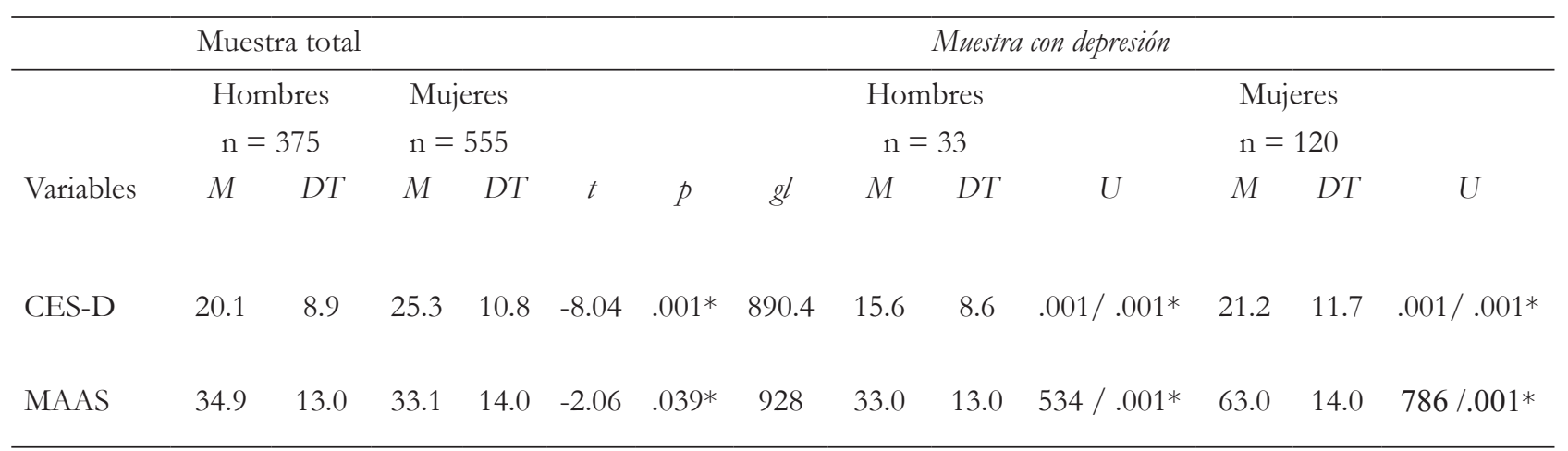

Nota: $\mathrm{M}=$ Media y DT $=$ Desviación Típica. ${ }^{*} p \leq 0.001$

Tabla 3

Asociación del CESD y mindfulness en cada sexo.

\begin{tabular}{|c|c|c|c|c|c|c|}
\hline & & & \multicolumn{2}{|c|}{ Mindfulness } & \multirow[b]{2}{*}{ Valor de $\chi^{2}$} & \multirow[b]{2}{*}{$p$} \\
\hline & & & Bajo & Alto & & \\
\hline \multirow{2}{*}{ Masculino } & \multirow{2}{*}{ Depresión } & $\operatorname{Sin}$ & 117 & 193 & \multirow{2}{*}{38.7} & \multirow{2}{*}{$.001 *$} \\
\hline & & Con & 52 & 13 & & \\
\hline \multirow[b]{2}{*}{ Femenino } & \multirow[b]{2}{*}{ Depresión } & $\operatorname{Sin}$ & 165 & 294 & \multirow[b]{2}{*}{81.9} & \multirow[b]{2}{*}{$.001 *$} \\
\hline & & Con & 83 & 13 & & \\
\hline
\end{tabular}

Nota. *Valor de $p<.001$ (Usando prueba $\chi^{2}$ ), al comparar hombres y mujeres con depresión y sin depresión.

depresión (SD) tanto en el grupo de los hombres como en el de mujeres, lo cual constituye, sin duda, un foco de alarma. Esto porque, además de las diferencias que se han reportado para cada sexo, se ha hecho también hincapié en que los hombres son menos propensos a manifestar sus síntomas por las condiciones socioculturales a los que se enfrentan (Amador, 2001). Por lo que se sugiere estudiar con mayor detenimiento el desarrollo de los $\mathrm{SD}$ en los hombres, así como la manifestación de los mismos porque probablemente se esté infravalorando el diagnóstico, impidiendo detectar oportunamente problemas relacionados a los trastornos afectivos.

Por otro lado, en los últimos años se ha cobrado gran relevancia al estudio de variables psicológicas positivas que permitan la disminución de la presencia de síntomas de depresión como es el caso de la atención plena o mindfulness (Marchand, 2016). Los datos presentados en este estudio corroboran la hipótesis inicial sobre la existencia de una asociación importante entre bajos niveles de mindfulness y la presencia de síntomas depresivos en adolescentes.

Lo anterior coincide con los resultados encontrados en diversas investigaciones (Calvete et al., 2014; LópezMaya et al., 2015; Milligan et al., 2016) donde se reporta una estrecha relación entre estas dos variables; por lo que se concluye que el mindfulness, además de ser un estado mental donde el individuo está consciente de sus emociones, enfoca la realidad del momento sin dejarse llevar por prejuicios o reacciones emotivas negativas (Martín-Asuero \& García, 2007). También, es posible que, manteniendo estos mecanismos, se reduzca el malestar psicológico a través de una auto- 
Tabla 4

\begin{tabular}{|c|c|c|}
\hline & \multicolumn{2}{|c|}{ Depresión } \\
\hline & $\mathrm{r}_{\mathrm{s}}$ & $p$ \\
\hline Mindfulness & $-.53 * *$ & $<.001$ \\
\hline
\end{tabular}

Nota. rs: Correlación de Spearman

$* * \leq .001$

regulación emocional donde se establezcan las causas y se inicien estrategias de afrontamiento para prevenir consecuencias negativas (Linares, et al. 2016).

Con respecto a la comparación de medias entre los síntomas depresivos y los niveles de mindfulness, se observa que tanto en la muestra total de adolescentes como en la muestra con síntomas de depresión existen diferencias estadísticamente significativas en ambas variables, siendo mayor el puntaje de mindfulness en hombres. En relación con los síntomas de depresión, las mujeres puntuaron más alto que los hombres, lo cual sugiere que, cuando los síntomas depresivos aparecen o se presentan, los niveles de mindfulness o conciencia plena disminuyen. Esto concuerda con lo reportado con Deng, Li y Tang (2014) y Linares et al. (2016): que cuando estaba presente el mindfulness, disminuían los síntomas depresivos.

En cuanto a las diferencias por sexo, los hombres obtuvieron mayor puntaje en mindfulness y las mujeres en síntomas de depresión; estas diferencias fueron estadísticamente significativas. Los resultados coinciden con lo reportado por Calvete et al. (2014) y difieren con López-Maya et al. (2015) y Sánchez y ArayaVargas (2014) quienes, luego de asociar las variables mindfulness y sexo, no encontraron diferencias entre las puntuaciones obtenidas por hombres y mujeres.

Lo anterior, permite sugerir que ambos hallazgos deben investigarse con amplitud para que, en un futuro, se puedan fundamentar ambos resultados sobre atención plena. Sin embargo, Matud, Guerrero y Matías (2006) explican que probablemente estas diferencias se deban a que, generalmente, son las mujeres quienes tienen más posibilidades de padecer síntomas depresivos a lo largo de su vida. Arguyen que se muestran más rumiativas ante los síntomas y los hombres cuentan con medios más eficaces para afrontar estados de ánimo negativos.

Otro hallazgo importante fue la asociación entre los síntomas de depresión y los niveles de mindfulness tanto en hombres como en mujeres, lo cual fue estadísticamente significativo. Estos resultados coinciden con los de Calvete et al. (2014) y apoyan la asociación negativa que existe entre mindfulness y síntomas de depresión.

Finalmente, al correlacionar las variables de mindfulness y depresión, se encontró que existe una correlación negativamente significativa entre ellas. Esto indica que, frente a puntajes bajos de mindfulness, mayor presencia de síntomas de depresión. Estos resultados concuerdan con lo reportado en estudios con adolescentes (Christopher \& Gilbert, 2010; Calvete et al., 2014) donde se menciona que el mindfulness o atención plena permite a la persona aprender sobre cómo funcionan, cómo detectar y cómo registrar sus pensamientos; cuál es la relación que tienen con emociones negativas y cómo puede aprender a cuidarse mejor (Segal, Williams \& Teasdale, 2013). De tal manera, tendrá la oportunidad de cambiar su experiencia a través de recursos psicológicos positivos como el mindfulness (Marchand, 2016; Remor, 2008).

Tomando en cuenta los resultados anteriores, donde se observa un incremento general en los síntomas de depresión en adolescentes, sin que esto se llegue a considerar un nivel clínico, estos síntomas sí tienen 
un efecto negativo en su rendimiento cotidiano a nivel físico, social y emocional. Por esta razón, es importante contar con especialistas dentro de las instituciones educativas para que puedan detectar a tiempo en los jóvenes problemas emocionales y puedan ser atendidos de forma oportuna para reducir la incidencia de trastornos emocionales y evitar que se agudicen en el futuro (Cortés et al, 2010; Ticusan, 2012).

Sin embargo, este trabajo presenta ciertas limitaciones; se trata de un estudio transversal, impidiendo determinar relaciones causales entre los síntomas de depresión y el mindfulness. Aunado a ello, la muestra de estudio fue de adolescentes estudiantes, dejando a fuera a los de la comunidad en general, posiblemente, con diferentes condiciones educativas, por lo que los resultados no se pueden generalizar.

En un futuro, se proponen investigaciones que incluyan a adolescentes de diversas condiciones culturales y educativas. Con esta recomendación, se puede dar fundamento empírico al papel del mindfulness como recurso psicológico positivo frente a los síntomas de depresión, además de estudiarlos con otros problemas emocionales.

\section{Referencias}

Amador, G. (2001). Género y depresión. Géneros 9 (25), 51-55. Recuperado de: http://bvirtual.ucol.mx/ descargables/421_genero_y_depresion.pdf

Barajas, S., \& Garra, L. (2014). Mindfulness and psychopatology: Adaptation of the mindful attention awareness scale (MAAS) in a spanish sample. Revista Clínica y Salud, 25(1), 49-56. doi: 10.1016/S11305274(14)70026-X

Barros, V., Kozasa, H., Souza, W., \& Ronzani, T. (2015). Evidences of validity of the brazilian version of the mindful attention awareness scale (MAAS). Psicologia: Reflexão e Crítica, 28(1), 87-95. doi: 10.1590/16787153.201528110

Benjet, C., Borges, G., Medina-Mora, M., Méndez, E., Fleiz, C., Rojas, E., \& Cruz, C. (2009). Diferencias de sexo en la prevalencia y severidad de trastornos psiquiátricos en adolescentes de la Ciudad de México. Salud Mental 32(2), 155-163.

Berenzon, S., Lara, M., Robles, R., \& Medina-Mora, M. (2013). Depresión: Estado del conocimiento y la necesidad de políticas públicas y planes de acción en México. Salud Pública de México 55(1), 74-80.

Brown, W., West, M., Loverich, M., \& Biegel, M. (2011). Assessing adolescent mindfulness: Validation of an adapted mindful attention awareness scale in adolescent normative and psychiatric populations. Psychological Assessment, 23(4), 1023-1033. doi: 10.1037/a0021338

Calvete, E., Sampedro, A., \& Izaskun, O. (2014). Propiedades psicométricas de la versión española de la escala de atención y conciencia plena para adolescentes (Mindful attention awareness scaleadolescents: MAAS-A). Behavioral Psychology: Psicología Conductual 22(2), 277-291.

Castro, A. (2010). Fundamentos de Psicología Positiva. Buenos Aires, Argentina: Paidós.

Christopher, M. \& Gilbert, B. (2010). Incremental validity of components of mindfulness in the prediction of satisfaction with live and depression. Current Psychology 29(1), 10-23. doi: 10.1007/s12144009-9067-9

Cortés, A., Aguilar, J., Medina, R., Toledo, J., \& Echemendia, B. (2010). Causas y factores asociados con el intento de suicidio en adolescentes en la provincia Sancti Spiritus. Revista Cubana de Higiene y Epidemiología, 48(1), 15-18.

Deng, Y., Li, S., \& Tang, Y. (2014). The relationship wandering mind, depression and mindfulness. Mindfulness, 5(2), 124-128. doi: 10.1007/s12671012-0157-7

Germer, C. (2011). El poder del mindfulness. Barcelona, España: Paidós.

Giménez, M., Vázquez, C., \& Hervás, G. (2010). El análisis delas fortalezas psicológicas en la adolescencia. Más allá de los modelos de vulnerabilidad. Psychology, Society \& Education, 2(2), 97-116. 
González-Forteza, C., Hermosillo, A., Vacio- Muro, M., Peralta, R., \& Wagner, F. (2015). Depresión en adolescente: Un problema oculto para la salud pública y la práctica clínica. Boletín Médico del Hospital Infantil de México, 72(2), 149- 155. doi: 10.1016/j. bmhimx.2015.05.006

González-Forteza, C., Jiménez-Tapia, J., Ramos-Lira, L., \& Wagner, F. (2008). Aplicación de la escala de depresión del Center of Epidemiological Studies de la Ciudad de México. Salud Pública de México, 50(4), 292-299.

Hankin, B., Mermelstein, R., \& Roesch, L., (2007). Sex differences in adolescent depression: Stress exposure and reactivity. Journal Child Development, 78(1), 279295. doi: 10.1111/j.1467-8624.2007.00997

Linares, L., Estévez, A., Soler, J., \& Cebolla, A. (2016). $\mathrm{El}$ papel del mindfulness y el descentramiento en la sintomatología depresiva y ansiosa. Clínica y Salud, 27(2), 51-56. doi: 10.1016/j.clysa.2016.03.001

López-Maya, E., Hernández-Pozo, M., MéndezSegundo, L., Gutiérrez-García, J., Araujo-Díaz, D., Nuñez-Gazcón, A., Cervantes-Sampayo, L., NavaAlcántara, S., Bautista, L., \& Hölzel, B. (2015). Psychometric properties of the mexican version of the mindful attention awareness scale (MAAS). Psychologia. Avances de la disciplina, 9(1), 13- 27.

Marchand, W. (2016) Mindfulness for the treatment of depression. En E. Shonin, W. Van Gordon y M. Griffiths (Eds.). Mindfulness and buddhist-derived approaches in mental health and addiction (139-164). doi: 10.1007/978-3-319-22255-4

Martín-Asuero, A., \& García, G. (2007). Las ventajas de estar presente: Desarrollando una conciencia plena para reducir el malestar psicológico. International Journal of Clinical and Health Psychology, 7(2), 369-384.

Matud, M., Guerrero, K., \& Matías, R. (2006). Relevancia de las variables sociodemográficas en las diferencias de género en depresión. International Journal of clinical and Health Psychology, 6(1), 7-21.

Milligan, K., Irwin, A., Wolfe-Miscio, M., Hamilton, L., Mintz, L., Cox, M., Gage, M., Woon, S., \& Phillips, M. (2016). Mindfulness enhances use of secondary control strategies in high school students at risk for mental health challenges. Mindfulness, 7(1), 219- 227. doi: 10.1007/s12671-015-0466-8

Miró, M., Perestelo-Pérez,L., Pérez,J., Rivero, A., González, M., De la Fuente, J., \& Serrano, P. (2011). Eficacia de los tratamientos basados en mindfulness para los trastornos de ansiedad y depresión: Una revisión sistemática. Revista de Psicopatología y Psicología clínica, 16(1), 1-14.

Nava, S., Araujo, D., Núñez, A., Hernández, M., Cervantes, L., López, E., Gutiérrez, J., Bautista, L., Hölzel, B., \& Méndez, L. (2015). Psychometric properties of the mexican version of the mindful attention awareness scale (MAAS). Psychologia. Avances de la disciplina, 9(1), 13-27.

Radloff, L. (1977). The CES-D scale: A self-report depression scale for research in the general population. Applied Psychological Measurements, 1(3), 385-401. doi: 10.1177/014662167700100306

Remor, E. (2008). Contribuciones de la Psicología Positiva al ámbito de la Psicología de la Salud y Medicina Conductual. En Vázquez, C. \& Hervás G. (Eds.), Psicología positiva aplicada. España: Desclée de Brouwer.

Rivière, A. (1991). Orígenes históricos de la Psicología cognitiva: Paradigma simbólico y procesamiento de la información. Anuario de Psicología 57, 129-155.

Sánchez, G., \& Araya-Vargas, G. (2014). Atención plena, inteligencia emocional, género, área de estudio y reporte de ejercicio en estudiantes universitarios costarricenses. Revista Iberoamericana de Psicología del Ejercicio y el Deporte 9(1), 19-36.

Segal, Z., Williams, J., \& Teasdale, J. (2013). Mindfulnessbased cognitive therapy for depression: a new approach to preventing relapse. 2 da. Ed. NY: The Guildford Press

Shih J. H., Abela J. R., \& Starrs C, (2009). Cognitive and interpersonal predictors of stress generation in children of affectively III parents. Journal of Abnormal Child Psychology, 37(2), 195-208. doi: 10.1007/s10802-008-9267-z

Smallwood, J., Fitzgerald, A., Miles, L., \& Phillips, L. (2009). Shifting moods, wandering minds: negative 
moods lead the mind to wander. Emotion 9(2), 271276. doi: $10.1037 / \mathrm{a} 0014855$

Ticusan, M. (2012). Low self-esteem, premise of depression appearance at adolescents. Procedia-Social and Behavioral Sciences, 69, 1590-1593. doi: 10.1016/j. sbspro.2012.12.103

Van den Hurk, P., Wingens, T., Giommi, F., Barendergt, H., Speckens, A., \& Van Schie, H. (2011). On the relationship between the practice of mindfulness meditation and personality- an exploratory analysis of the mediating role of mindfulness skills. Mindfulness, 2(3), 194-200. doi: 10.1007/s12671-011-0060-7
Vázquez, C., \& Hervás, G. (2008). Salud positiva: Del síntoma al bienestar. En Vázquez, C. y Hervás G. (Eds.), Psicología positiva aplicada. España: Desclée de Brouwer.

Veytia-López, M., González-Arratia, N., Andrade, P., \& Oudhof, H. (2012). Depresión en adolescentes: El papel de los sucesos vitales estresantes. Salud mental 35(1), 37-43.

Williams, J., Teasdale, J. Segal, Z., \& Kabat-Zinn, J. (2007). The mindful way through depression. New York: Guildford Press.

Recibido: 29 Abril de 2016

Aceptado: 12 Setiembre de 2016 